\title{
Visual Culture in Educational Environment and Innovative Teaching Technologies
}

\author{
E. A. Makarova \\ Humanities Department, Taganrog Institute of Management and Economics, Russia
}

Copyright $(2016$ by authors, all rights reserved. Authors agree that this article remains permanently open access under the terms of the Creative Commons Attribution License 4.0 International License

\begin{abstract}
Modern social environment is characterized by dramatic growth of information flows that are required to be processed to keep pace with the development of science and education. The information flows increase so rapidly that they are getting harder and harder to process using traditional methods. Visual thinking is a new way to deal with "the image of the world" not divided into separate ideas, but with holistic visual images created via visual thinking and visual operations. The goal of visualization in education is to transform huge volumes of data into graphic images easy for perception and memorizing. Visual images can range from detail graphic representations to abstract schemes and mind maps. Visual reality is presented as information constructs subject to "reading" and interpretation as much as any verbal text. Visual reality is not considered as of secondary importance in culture and education; it combines thinking and imagination thus creating semantic images unique in their cognitive potentials and in quality of multilevel information coded in them. After different teaching technologies are compared, it is concluded that visual ways of information representation are more effective because of their structured and systematized form that makes it easy to teach and learn. Such structures are kept in long-term memory and easily reached if necessary, thus contributing to memorizing a lot of information.
\end{abstract}

Keywords Visualization, Schema Theory, Innovations, Teaching Technologies, Mind Map, Omega Map, The Image of the World

\section{Introduction}

Visualization "has been shown to help learners learn, researchers create new knowledge, administrators to better structure and manage organizations, writers to write, and evaluators assess learning" [1] Objective reality exists independently of the individual perception or will. Of course, people are part of the world, along with their thoughts, feelings, attitudes, actions; we do not live in the space of things, but in the space of events. These events are reflected in the visual images and verbal texts with the help of which people usually describe them. According to J. Piaget, knowledge of the world is not discovered, but created as an image. "Any image is a subjective picture of the world or its fragments, subjective representation of outside world objects due to both the sensible signs and hypothetical constructs" [2]. Since the image is rather a scheme (a frame), the human psyche is not the way, namely semantic world, created by the power of human imagination, it "includes the individual, other people, the spatial environment and temporal sequence of events. As the foundation for practical action for the exploration of the outside world, the image is also determined by the nature of these actions, during which the original image is modified, all the more satisfying practical needs. "In his intellectual development the individual goes from roughly semantic search - emotionally rich and expressive - to actions of creating images. The image contains the model of the world in its semantic shape and suggests semantic integrity. Semantic integrity is the perfect environment within which all subjects, qualities and properties of the material world are considered from a certain point of view and are interpreted based on the practical experience. The image includes the creation of personal meaning (the successive formation and semantic content of personal meaning as a way of structuring and organizing) and the author's participation in the act or event. Personal qualities included in the semantic world are correlated not only with knowledge but also with self-reflection.

Modern society is characterized by a dramatic increase of information flows that people need to process in order to maintain progress in the development of science and education. The volume of information is growing so fast that people are no longer able to comprehend amounts of numerical or factual data and to process those using traditional methods. The basis of the "image of the world" lies not in the concept itself, but in a semantic image created via visual thinking. Visual thinking is a kind of mental operations that can be used to analyze data, it leads to a purely human ability to see and understand the images, "the representation of knowledge in the form of structures in 
motion; . . . the study of relationships of these forms and structures; . . . the flow of images as pictures, diagrams, explanatory models, orchestrated paintings of immense ideas, and simple gestures; ... work with schemes and structures of the mind" [3]. "Visual thinking may contribute to understanding in more than one way. It includes thinking with external visual representations (e.g., diagrams, symbol arrays, and kinematic computer images) and thinking with internal visual imagery; often the two are used in combination, as when we are required to visually imagine a certain spatial transformation of an object represented by a diagram on paper or on screen." [4]

Accordingly, the task of visualization is the conversion of huge amounts of information in an adequate readable symbols associated with semantic image. Visual images may vary from the detailed graphics to abstract structures.

\section{Visual Reality and Graphics}

Visual reality is presented as an information construct, to be 'read' and interpreted to the same extent that these procedures are done to verbal text. This reality is no longer perceived as a secondary or subordinate dimension of cultural practices, but as a combination of semantic thinking and imagination. As a source for the study, the visual material is unique in its cognitive and multilevel potential to code information. Comparative analysis of different methods of presentation of information leads to the conclusion that visual methods are more effective, because they represent information in a more structured and systematic way and make it more visible. Graphics helps visualize and understand in order to shape the information structure, moreover, the human brain always looks for symbols to present the information. Using schemes increases motivation and flexible, critical thinking. Images and ideas are combined to enable development of logical thinking. Visualization is a way to keep and transform information, not only for current use, but also for future challenged dominantly verbal and written communication. At present, the "doubling of the cultural environment" in which all the achievements of mankind, fully reflected in the previously written texts, audio-visual expression is obtained (visualization of culture). Visualization compared with verbal communication is psychologically more concise and easier, but less systematic and rational, socially more widespread and affordable, but less stable and unidirectional way of structuring knowledge. These features form the visualization in its socio-cultural (communication) and cognitive function (teaching and learning).

The communicative function of visual images is rapidly becoming a leading means of mass communication (in the form of television, multimedia, etc.). Taking on the related socio-regulatory responsibilities and acquiring proper forms and shapes this function is engaged in political and ideological activities, organizational and economic ties, which have a serious impact on its further development. The reasons for this are many, because in the world there is a global shift from the verbal to the visual component. In modern society, the bombardment of visual images (such as television images, video, television advertising, computer images, signs, symbols, icons, logos) has such an impact on human mentality that people are becoming less able to perceive the written word and cannot focus on the verbal text for a long period of time. Visualization permeates all spheres of life.

J. Mitchell (one of the modern scholars of visual studies) noted that in recent years there has been a real revolution in science, coupled with an interest in the study of visual culture. We are talking about investigations of film, television, commercials from the position of modern theories to explain the specifics of 'visual society'. This is especially true of education and brings to life the need to create an auxiliary visual support and visual organization for the study of educational material [5].

Despite the increasing application of didactic means, involving a combination of information impact on various human senses (hearing, touch and even smell), for presenting educational information, visual is the leading type of perception, which involves the development of both traditional and innovative tools and techniques that enhance the work of the learning process. Gradually, the need for innovation is becoming more evident and requires a speedy judgment because education in our country, as in all other countries of the world, at the beginning of the XXI century entered a crisis situation, not being able to get out of it, utilizing well-established traditions and teaching methods. Thus, we can describe the traditional didactics crisis: the old techniques and technologies of teaching cannot be applied, but a new way to effective teaching has not yet been obtained. Both looking for new didactic principles of training based on information technology, (and perhaps new didactics) are based on the formation of semantic way to create "image of the world". A visual image is a mental image that is similar to a visual perception, an image in the visual system.

Using visual analyzers requires taking into consideration the laws of visual perception and proper use of visual methods in teaching. Therefore, with, particular structuring, coding, and decoding, the amount of teaching material can be minimized. Tools of visualization are used to transfer large amounts of information, to update cognitive mechanisms that complement the verbal channels of information. Such structured data arrays represent a new generation of educational materials in which image and text are connected organically and mutually reinforce each other, thus achieving a synergistic effect. Synergy comes from the Greek word "synergy" meaning "cooperation" and focuses on the consistency of the interaction part in the formation of the structure as a whole. Synergy, as a scientific concept of holistic perception of the world and individual systems is shown in the combined action of components of any self-organizing system. The basic idea of these materials is to convey a thought in the unity of image and text. Figurative and verbal components of thinking alone are not as strong as 
in unity, in addition, a great teacher teaching semantic value obtains the unity of the verbal and the visual components. Visualization in teaching is based on the special properties of mental images as objects of knowledge, expresses the degree of accessibility and clarity of these images for the subject. It relies on one of the most important principles of learning (the principle of clarity). Visual images are divided into sensory aids and rational ones (in abstract form reflecting the most common and essential aspects, communication and relations of the objective world, inaccessible directly by senses). By creating an image a learner comes up to solution of cognitive tasks, which, along with the senses involves memory, thinking, imagination and personal meaning.

\section{Image of the Inner World of a Person}

Any external reality is reflected in the framework of a model in the inner world of the person created by a specific activity, expressed in the process of perception. A person gets all the information perceived from the outside, first in the form of separate primary sensations, but all he sees, hears, touches is refracted through the prism of understanding and awareness, and only then becomes some mental construct, a way of reality. Becoming internal this information acquires appropriate personal meaning and becomes a mental construct.

Internal and external resources are closely interrelated. Any external means may be fixed in the form of images, models, descriptions and can be internalized through active reflection of consciousness. Internalized external agent is not some kind of a copy placed in the consciousness of the person, but it includes many additional elements and connections between them, due to knowledge, peculiarities of the psyche and mental processes actualized by the person. Consequently, internalized external agent is a sufficiently independent phenomenon, although generated by external means. In addition, the composition may contain internal means or means not directly connected with the external object actions. It can be concluded that the system of internal funds of the person used for training is much richer, more diverse and more complex in structure than the system of its external assets. On the basis of this conclusion, it can be assumed that the development of teaching aids should go towards updating the internal funds. One method is a didactic simulation (creating mental models, frames, diagrams) as the best and the most economical way of keeping scientific knowledge. Modeling any external object (external phenomenon, process, etc.) can be represented as a different object (internal) for the purposes of study which serves as a means to obtain knowledge about the original object. The method of modeling is visualization.

Works such as Temple Grandin's Thinking in Pictures [6] and Thomas G. West's In the Mind's Eye [7] have revealed how crucial it is for teachers to cultivate visual thinking and adjust their methods for visually inclined students. New research indicates that we need to move beyond categorizing people as "visual" or "verbal" and consider the many different mental processes that visual thinking involves. "A rule of the game" in such teaching, visualization should represent educational content (an idea, information content (static) or dynamic (multimedia) training tools). This content should be based on a constant interaction of three methods of presentation of information (text, image and diagram), resulting in a holistic training, allowing the trainee to develop visual thinking in the study of subjects of different educational spheres. The importance of the latter is indicated by an American psychologist Rudolf Arnheim, "The best intentions a biology teacher may have, will hardly be perceived by inadequately prepared students, if the same principle is not applied by the math teacher" [8].

\section{Visualization for Teaching Purposes}

Thus, the basis for visualization of the teaching materials content is in conscious and purposeful use of educational "gestalt", specially designed and organized in a special way to stimulate the perception of educational material and to develop visual thinking. The rationales for this approach are the three statements: it is easier to navigate in figural material, it is better remembered and as the hallmark of this unique training program makes it easy to distinguish from the others, and then instantly restores the entire structure in memory at any mention of it. Both natural and artificial objects generate stable semantic images often accompanied by certain emotions in person's mind, called 'gestalts'. "Gestalts" are always wholes, each of which, in its turn, are a part of the whole integrity of consciousness with symptoms of narrow-mindedness and ruggedness, and total integrity of consciousness and is a complete structure consisting of a variety of components more or less distinct, but related to the whole. Studies of Gestalt psychologists have created new approaches to various problems - from creative thinking to the personal activity. Multiple studies of the psyche have made it possible to understand the laws of perception, thinking and personality development to form fundamentally different from previous experimental procedures.

Gestalt ideas are used particularly in relation to schemes in the framework of visual perception studies. "In addition, or instead, purely visual images there are also common types or schemes in terms of how the person designed answers. With time passed the schema itself becomes even more dominant, initial visual images disappear, details contained in the original are forgotten and reproduced incorrectly, even though the last representation usually shows a steady movement to the image of the type or scheme, which was originally intended". [9]

Back in the 1970s, the cognitive style categories "visual" and "verbal" were set up as opposites. Not only do "visual" and "verbal" thinking lump diverse skills; they coexist in every mind, and creative impulses emerge when they interact. Being visually inclined may mean being able to describe and analyze images with words. [10] 
It seems that adequate visualization should first convince the student (only on an intuitive level) in the truth of certain statements. Therefore, the use of graphic images should be particularly useful on the stage of concept development. By a concept we mean a symbolic display of the essential properties of objects of the world, selected as a result of the analytical work. Each concept includes a particular action, reproducing the object of knowledge through the use of certain tools. What mental images, diagrams generate certain kinds of visualization? How sustainable are they? How do they interact with each other? What is the interjection? Is it semantic? These are the questions that require combined studies by both teachers and psychologists.

Introjection is the inclusion of perceived attitudes, motives and attitudes of others; identity-based, psychological mechanism into an inner world by an individual; the process that plays an important role in the shaping and storage of knowledge in memory, epistemological concept that individual consciousness is limited by the terms of their own ideas and is able to comprehend the outside world and another person's consciousness only by projecting the outside reality into their inner content. Sometimes introjection is called "positive transference"; the concept itself is close to interpenetration.

Introjection is semantic and can encourage greater degree of comprehension, generalization of perceived images, specify and improve the completeness, integrity of images and ideas. Visual representations can develop audio training that is fundamental, but purely verbal. Using visual techniques in teaching allows learners to use several types of memory along with verbal logic, including the mechanisms of visual-figurative and emotional memory. What is the main feature of introjection in training (compared to traditional options) and its significance for integration in education? What are the specifics of this knowledge? From the psychological point of view, the main thing in this issue is that introjection generally blurs the traditional modality of knowledge. Instead of a value system learnt by studying mathematics, physics, biology, history, etc., learners begin to comprehend the conceptual system rather on the level of the system knowledge than on the level of a certain science, i.e., knowledge itself becomes multimodal. Presentation of information in the form of multimodal knowledge stimulates long-term preservation of information in the memory, increases the accuracy of memorized information, thus increasing the effectiveness of memorizing. Thinking processes using the visualization training methods can also be strengthened by expanding the kinds of thinking activated. Along with the abstract logic, visualization of information allows learners to include mechanisms of visual efficient, imaginative, associative thinking, to enhance the activity of the imagination (creative and re-creative thinking).

Multimodal knowledge may be a part of mental content only if it is formed through student's internalization of components by cognitive mechanism and creating images. And it becomes possible to achieve with any integration (because it is clear that integration can take place on the level of value integration), but only in the integration-oriented introjection into mental space.

In the experimental cognitive psychology existence of mental space (environment) is confirmed by experiments studying organization of semantic memory as well as the processes of understanding the text (which involves creating the content of text in the mind space and complex operators for mental movements in this space). One of the first concepts of mental space as a psychological category was introduced by G. Fauconnier [11], who used it in a fairly narrow context of knowledge representation. Inside this space, there are mental objects and combined mental connection. Subsequently, the concept of mental space was used by B.M. Velichkovsky [12] to explain the effects of information processing on the level of the highest symbolic functions. Mental spaces are small conceptual packets constructed as we think and talk, for purposes of local understanding and action. They are very partial assemblies containing elements, and structured by frames and cognitive models. They are interconnected and can be modified as thought and discourse unfold. Mental spaces offer a general and elegant means of dealing with opacity, presupposition, counterfactuals, and tense and mood in language. Thus, it was shown that the presentation of the real space unit can be deployed immediately into a full mental spatial context depending on the task.

\section{Visualization as Inner Mental Representation of the Outer World}

Despite this attention by psychologists, mental space is a mental phenomenon that has yet to be the subject of a detailed psychological study. However, it is the concept of "mental space "that is the missing theoretical link that allows moving from the concept of "mental structure" to the concept of "mental representation". Each mental space defines its own semantic context, has its own emotional background and dictates the rules of construction activities. Each person describes the mental space of own reality - the reality of human performance, whether it is a memory of the past or dreams for the future, reconstruction of the historical event or self-image.

Psychological basic visualization tools can be classified as:

- cognitive mechanisms of actualization (activation of perception, memory, thinking, imagination, etc.);

- development of mental functions (motivation, attention, observation, aesthetic perception, etc.);

- psychological impact on (creating an emotional background of perception, relaxation, attitude to learning and so on.);

- continuously updated cognitive functions (ideas, concepts, information modeling, coding and decoding and others);

- different theories of assimilation (a step by step 
action, associations, Gestalt perception, holistic learning).

Understanding is a multi-level procedure where thinking is only one of the levels. We cannot prove that understanding is impossible without seeing, but it is quite difficult. The point is the detection value of the result of detection is to understand the meaning. A person can consciously perceive the objects in the perceptual field and at the same time think about these things that can be confined to the actual perceptual space or be phenomenological unobservable objects in general. In this case, one can speak of a parallel, independent of one another actualization area meanings representing the consciousness of the subject at the same time through a variety of educational paths, which should lead to the conclusion that consciousness (in terms of understanding) is the ability to "read" multiple texts (verbal or visual). Thus we overcome the methodical discrepancy in educational process and solve a problem that has traditionally been the most difficult one in educational psychology and didactics.

Multimodal knowledge may be a part of mental content only if its internalization occurs through multimodal components of cognitive mechanism - through student's making sense and getting to understand the meaning. And it becomes possible to do with any integration (because it is clear that it can take place at the level of integration of values), but only in the integration-oriented introjection. Meaning, unlike values, is always multimodal and thus forms the basis of the psycho-semantic image mediating real mental activity. This suggests that a student can log in integrated education, but on a personal level. This approach determines the view of thinking (including visual thinking) as of the action or activities of the mind, whereby it becomes possible to understand the connections and relationships between the studied objects in the study of learning content in a modern information educational space.

The introduction of information technology identifies new trends of teaching materials visualization as the most appropriate and efficient ones in perception, processing and memorizing information. The practice of teaching highlights several areas of educational content visualization, which are examples of the educational activities organization:

- representation of the new educational information as illustrations, pictorial representation,

- in the form of charts, tables, graphs, drawings, graphics, images, etc.;

- fixation of teaching material in the form of text or graphic, as well as the systematization of computer graphics and computer set pieces;

- preparing students for the preservation of intellectual property, independent, creative activities for the acquisition of knowledge, including ones through implementation of computer graphics;

- interpreting educational information in accordance with the terms and scenario training session, the transformation of the educational content in scheme, frames, constructs convenient for long-term storing.

A fundamentally new problems can be solved in education systems using computer modeling, expert systems, artificial intelligent and so on. In all these tasks a special place and role belongs to imaging techniques. The use of information technology has brought new principles into the classification of visualization. Methods of visualization in teaching information technology can be considered as follows:

- passive (processing of still images, pictures, graphs, diagrams etc.);

- active (working with a database, virtual libraries, participation in e-forums, virtual round tables, teleconferences, webinars, e-learning etc.);

- combined (work with the Internet, electronic books, entertainment and educational software products (edutainment), creating projects and presentations in PowerPoint).

To some extent, this division can be regarded as conditional, but nonetheless, it helps to understand the importance of visualization in educational activities. Selection of visualization techniques for educational purposes as a basis for work in the information environment is important to prepare students to respect intellectual property and to protect intellectual property both affecting formation of communicative culture in its aesthetic, ethical, informational aspects. The communicative culture and the attitude to the acquired information increase with the experience expansion in the process of training. It is important to remember that visualization is a complex psychological process that has an impact on the development of visual memory, associative, imaginative and logical thinking of students, on the formation of semantic meanings.

\section{Visual Methods in Education}

Information technology equipment and specially designed software products open up new possibilities in developing students' necessary skills and abilities (perceptual, mental, verbal, practical and so on). When rendering educational content, not only common but differential psychological characteristics, possible types of students' cognitive activity, different types of major sensory modalities and other psychological peculiarities should be taken into account. Visual methods in learning can solve a variety of educational objectives: the development of observation, visual memory, creative thinking and so on.

Special methods of educational activity management can be applied with the help of visualization tools that has an impact on the students' activity, their self-regulation and self-activation in the learning process. When imaging techniques are competently used the students' transition to higher levels of cognitive activity is stimulated by the mastery of subject content with elements of creativity and heuristics. But most importantly - a new informational and educational environment is created, and fundamentally new 
roles of the teacher (as a consultant, trainer, coordinator, and moderator) and the student (independent researcher and independent learner) support a new relationship between the two. The rapid development and implementation of information technology in the educational process leads to a radical rethinking of the traditional objectives, content, forms and methods of teaching.

Psychological foundation of visualization tools application can be classified as follows:

- cognitive mechanisms of knowledge actualization (activation of perception, memory, thinking, imagination, etc.);

- mental functions development (motivation, organization of attention, development of observation, aesthetic perception, etc.);

- psychological impact (emotional background of perception, relaxation, attitude to work and so on);

- continuous update of cognitive functions (formation of ideas, concepts, information modeling, information coding and decoding, and others.).

- different theories of assimilation (the formation of a step by step action, the formation of associations, the Gestalt perception).

In order to understand how disparate elements of reality are combined into a holistic mental construct (semantic meaning) presented in the consciousness of a student, it is necessary to study the system of mental images which reflect not only a person's representation of the outside world, but also his/her inner world, it should include personal meanings and be meaningful for the individual. Active ownership of the information is possible only when the essential properties of the image of the world are structured and clearly explained.

Mental structures formed by human intellectual resources play the main role in the process of creative thinking. As logic is based on verbal thinking, so intuition is based on creative thinking. Creative thinking is no less important than the verbal one, moreover, the figurative and verbal components of thinking alone are not as strong as united. Mental structures are the basis of individual mental experience. They form a system of mental concepts, which in cognitive contact with reality allows the transformation of information flow, as well as the process of information control, its processing and selectivity of intellectual reflection.

In their study cognitive neuroscientist M. Kozhevnikov and her colleagues have found evidence that people who think visually rely on two distinct groups of skills. Those who lean toward object visualization excel at picturing scenes in rich detail and distinguishing shades of color. They often have excellent visual memories and process scenes holistically. Those inclined toward spatial visualization do well at judging distances, relative dimensions, and velocities and often process ideas in terms of schematic patterns [13].

Failures of intellectual activity may be an indication not so much of a lack of intellectual gift, but of the inability to express the available mental capacity. In different definitions it is emphasized that mental structures have specific manifestations of intellectual activity and depend on individual features and characteristics of human social behavior.

In other words, mental structures are a kind of psychological mechanism which can "deploy" intellectual resources when confronted with any external influence in a specially organized mental space. Mental images are often called "quasi-perceptual experience" and greatly resemble real perceptual experience, but in the absence of perceptual stimulus. They are not associated with imagination and fantasy, but with such cognitive functions as memory, perception and thinking. Although mental images occur in all sensory modes, most of the publications in the field of philosophy, psychology and pedagogy are only focused on visual images.

J. Lakoff and M. Johnson offer imaginative schemes as opposed to verbal schemes, which they explained as the embodiment of "unsaturated" form of images, produced by imitation of earlier images, and widely applicable to various stages of the perception process, so that they reproduce a very wide range of subject classes. Being schematic, these images provide a "metaphorical" support for a wide range of specific mental processes. Such schemes do not have the richness of detail, but although they lack in quality and richness of the direct impact on the mind, they make up for the expense of generalization and the functional significance of thinking [14].

At present, psychologists have not yet achieved a complete understanding of the visualization value as the educational environment necessary for communication development. However, the foundations for scientific knowledge representation required for each student have already formed the basis of visualization, maintained and developed by psychological and pedagogical technologies, by philosophy, culture study and other sciences $[15,16]$.

\section{Conclusions}

To conclude and to make it clear, we do not believe that visualization (charts, frames, Gestalt and art images) can entirely replace a verbal method of presenting information. However, the visualization of the information content (together with a personal meaning and the semantic image) will direct thinking in the desired direction towards the most important integration of visual and verbal methods of information presentation thus helping students comprehend the reality of the world and build their own "image of the world" adequate to this reality.

During implementation of visual techniques and technologies in teaching and learning psychological characteristics of cognitive activity mechanisms must be taken into consideration. It is important to be aware of the ratio of words to images in the visual teaching materials. Visual learning techniques help solves a variety of 
educational problems: the development of observation, visual memory, creative thinking and so on. The imagery as a method of information transfer creates prerequisites for the learners' development as a whole and particular mental process.

Due to the growing interest in the visual material, it is necessary to study the peculiarities of the perception, the study of the specifics of making sense of images and transforming them into consciousness to build a constructive communication between a teacher and a learner.

In order to understand how disparate elements of reality are combined into holistic mental constructs, presented in the consciousness of the learner, it is necessary to study the system of mental images, which is reflected not only by the person's representation of the outside world, but also their attitude to this world. To achieve this, image of the world formed by a person should involve personal meanings thus creating the interest and attitude.

Visual thinking is getting more popular in different spheres of life, so we can speak about visual culture being developed. "Visual culture is concerned with visual events in which information, meaning or pleasure is sought... Visual culture does not depend on pictures but on this modern tendency to picture or visualize existence."[17]

\section{REFERENCES}

[1] Novak Joseph D. \& Alberto J. Cañas (2006) "The Theory Underlying Concept Maps and How to Construct Them." Florida Institute for Human and Machine Cognition.

[2] Piaget, Jean (2004) Psychology of Intellect. St. Petersburg Piter.

[3] John-Steiner, Vera (1985) Notebooks of the Mind: Explorations of Thinking. New York: Oxford University Press, 109.

[4] Giaquinto, Marcus (2015) “The Epistemology of Visual
Thinking in Mathematics”, The Stanford Encyclopedia of Philosophy (Winter 2015 Edition), Edward N. Zalta (ed.), $\mathrm{URL}=$ http://plato.stanford.edu/archives/win2015/entries/epi stemology-visual-thinking/

[5] Mitchell W.J.T. (2002) Showing Seeing: a critique of visual culture. Journal of Visual Culture. (Vol. 1. No 2. pp. 165-181).

[6] Grandin, Temple (2006) Thinking in Pictures and Other Reports from My Life with Autism. London: Bloomsbury.

[7] West, Thomas G. (2009) In the Mind's Eye: Creative Visual Thinkers, Gifted Dyslexics, and the Rise of Visual Technologies. Amherst, NY: Prometheus Books.

[8] Arnheim R. (2007) Art and Visual Perception. M.: Architecture.

[9] Wulf Friedrich (1941) Tendencies in figural variations. F. Wulf.//A Source Book of Gestalt Psychology/Ed. BY W.D. Ellis. N.Y.: Harcourt-Brace, p. 75.

[10] Kozhevnikov, Maria, Olesya Blazhenkova, and Michael Becker (2010) "Trade-Off in Object versus Spatial Visualization Abilities: Restriction in the Development of Visual-Processing Resources”. Psychonomic Bulletin and Review 17.1: 29-35.

[11] Fauconnier, Gilles (1985) Mental spaces. Cambridge.

[12] Velichkovsky B.M. (1982) Modern cognitive psychology. M.: MSU.

[13] Kozhevnikov, Maria, Stephen Kosslyn, and Jennifer Shephard (2005) "Spatial vs. Object Visualizers: A New Characterization of Visual Cognitive Style." Memory and Cognition 33.4: 710-26.

[14] Lakoff, G. and M. Johnson. (1980) Metaphors We Live By. Chicago: University of Chicago Press.

[15] Pollock G. (2001) Contemplation on the history of art: vision, position and power. Gender Studies. Tutorial Part 2 (HZGI). (pp. $718-737) . S$ P.: Aleteia.

[16] Andrews J. (2014) Showing Off! A Philosophy of Image. Bloomsbury Academic, London.

[17] Mirzoeff, Nicholas (2008) An Introduction to Visual Culture. London and New York: Routledge, $2^{\text {nd }}$ ed. 